\title{
Characterization of Meta-Materials Using Computational Electromagnetic Methods
}

\author{
Manohar Deshpande*, NASA Langley Research Center, Hampton, VA., USA \\ m.d.deshpande@larc.nasa.gov \\ and Joon Shin, NRC Research Associate, NASA LaRC, Hampton, VA., USA
}

\begin{abstract}
An efficient and powerful computational method is presented to synthesize a meta-material to specified electromagnetic properties. Using the periodicity of meta-materials, the Finite Element Methodology (FEM) is developed to estimate the reflection and transmission through the meta-material structure for a normal plane wave incidence. For efficient computations of the reflection and transmission over a wide band frequency range through a meta-material a Finite Difference Time Domain (FDTD) approach is also developed. Using the Nicholson-Ross method and the Genetic Algorithms, a robust procedure to extract electromagnetic properties of meta-material from the knowledge of its reflection and transmission coefficients is described. Few numerical examples are also presented to validate the present approach.
\end{abstract}

\section{Introduction}

In the recent years there has been a great deal interest in studying the material (called meta-material [1-3]) having simultaneously negative permittivity and permeability. These meta-materials do not exist in nature and are artificially formed by embedding properly designed metallic/non-metallic inclusions in binding mediums. In such a

material, the wave vector $\vec{k}$ and the Poynting vector $\vec{P}$ are anti-parallel causing reversal of some basic electromagnetic wave propagation characteristics such as Snell's law and Doppler effects. The reversal of these basic propagation properties has lead to some potential applications of these materials such as high resolution lenses,

sub wavelength resonators and enhancement of antenna gains. One of the challenges faced by the researchers in this field is lack of a robust method to characterize these materials. The characterization of the meta-materials is carried out in two steps. First, the reflection and transmission properties of these materials are estimated /measured using a computational/experimental method. In the second step, a proper inversion process is used to extract effective electromagnetic properties from the knowledge of reflection and transmission coefficients. Experimental procedures are too expensive and time consuming to explore all design space, and are is usually not preferred in early stages of design cycle. In the present paper, an efficient and powerful computational method is presented to characterize/estimate effective electromagnetic properties of meta-materials. In this method, first, the metallic inclusions of interest are modeled using a commercially available Computer Aided Design (CAD) package. Using the Finite Difference Time Domain method the reflection and transmission through these inclusions are then estimated. In the final step using an inversion procedure based on two approaches, Nicolson-Ross method [4] and Genetic Algorithm, effective properties of the selected inclusions are extracted. Few examples with known effective properties are presented to validate the present method.

\section{Analysis of Meta-Materials}

In this section, the development of computational methods based on the Finite Element Methodology and also Finite Difference Time Domain (FDTD) method are presented. Figure 1(a) shows a metallic inclusion in a split ring form and Figure 1(b) shows a dielectric medium embedded with a periodic arrangement of split ring shown in Figure 1(a). It is assumed that the structure is infinite in the $x-y$ plane and has finite width in the z-direction. It also assumed that the structure is illuminated by a plane wave with normal incidence as shown in Figure 1(b). 


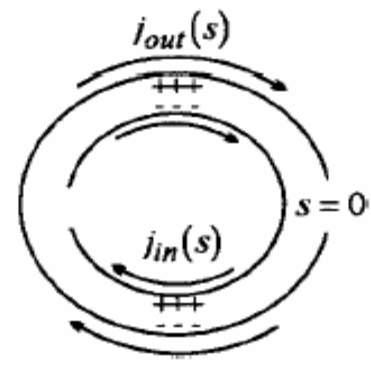

Figure 1(a) Split Ring

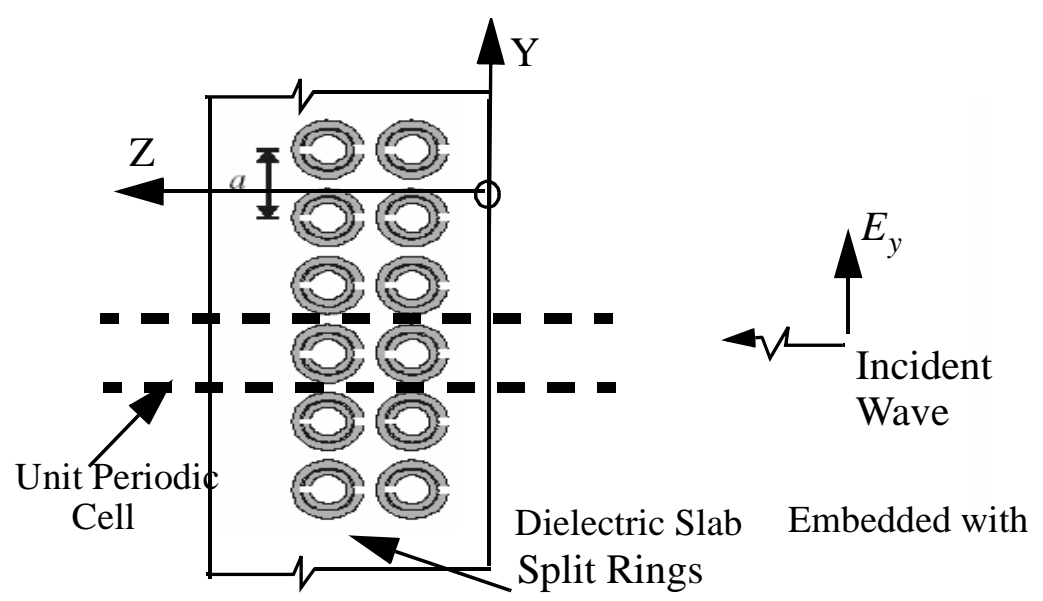

Figure 1(b) Dielctric slab embedded with split rings

Finite Element Method: To estimate the reflection and transmission coefficients of such a structure, a unit periodic cell as shown in Figure 1(b) is considered. Using the electric and magnetic symmetries, the unit cell can be terminated by electric and magnetic conducting boundaries at $y= \pm a / 2$ and $x= \pm b / 2$, respectively. The electromagnetic field incident at the input port of the unit cell can be written as

$$
\vec{E}_{\text {in }}=\hat{y} E_{0} e^{-j k_{0} z}, \quad \vec{H}_{i n}=-\hat{x} E_{0} / \eta_{0} e^{-j k_{0} z}
$$

The electromagnetic field scattered in the regions $z \leq 0$ and $z \geq d$ can be expressed in terms of the Floquet modes. Using the FEM formulation, the weak form of wave equation to be solved for the electric field in the unit cell takes following form [5]

$$
\begin{aligned}
& \int_{V}\left(\nabla \times \vec{T} \bullet\left(\frac{1}{\mu_{r}} \nabla \times \vec{E}\right)-\left(k_{0}^{2} \varepsilon_{r} \vec{T} \bullet \vec{E}\right)\right) d v=2 j \omega \mu_{0} Y_{0} \int_{S_{1}} \vec{T} \bullet \vec{E}_{i n} d s \\
& -\left.\left(j \omega \mu_{0}\right) \sum_{p=1}^{\infty} Y_{p} \int_{S_{1}} \vec{T} \bullet \vec{e}_{p}(x, y) d s \int_{S_{1}} \vec{E}^{I I}\right|_{S_{1}} \bullet \vec{e}_{p}(x, y) d s-\left.\left(j \omega \mu_{0}\right) \sum_{p=1}^{\infty} Y_{p} \int_{S_{2}} \vec{T} \bullet \vec{e}_{p}(x, y) d s \int_{S_{2}} \vec{E}^{I I}\right|_{S_{2}} \bullet \vec{e}_{p}(x, y) d s
\end{aligned}
$$

where $\vec{E}^{I I}$ is the electric field in the unit cell volume $V$ bounded by $z=0$ and $z=d$ planes, $\vec{e}^{p}$ is the vector modal function for the Floquet modes, $Y_{p}$ is the modal admittance for the $p^{t h}$ mode, $\vec{T}$ is the testing function, and $S_{1}, S_{2}$ are the surface areas at the $z=0$ and $z=d$ planes, respectively. From the solution of equation (2) the reflection and transmission coefficients for the structure shown in Figure 1(b) can be determined.

FDTD Method: It is often required to estimate the reflection and transmission of EM waves through meta-material over multiple frequencies. The FEM approach presented above becomes computationally intensive when required to be repeated for each frequency of interest. The FDTD method provides an alternative and efficient way of estimating reflection and transmission through meta-materials over multiple frequencies. To perform these computations using the FDTD approach we used the commercially available CST Microwave Studio [6] computational tool. To validate the FEM code and the numerical results obtained using the CST software, a dielectric slab embedded with four split rings and capacitively loaded strip dipole as shown in Figure 2(a) is considered. The reflection and transmission coefficients obtained using both approaches are shown in Figure 2(b). The close agreement between the two numerical 
results confirms validity of the FEM formulation and the also the modeling procedure followed while using the CST Microwave Studio.

Using these techniques the reflection and transmission characteristics of metallic inclusions of various shapes and sizes are computed and results will be shared with the audience at the time of presentation.

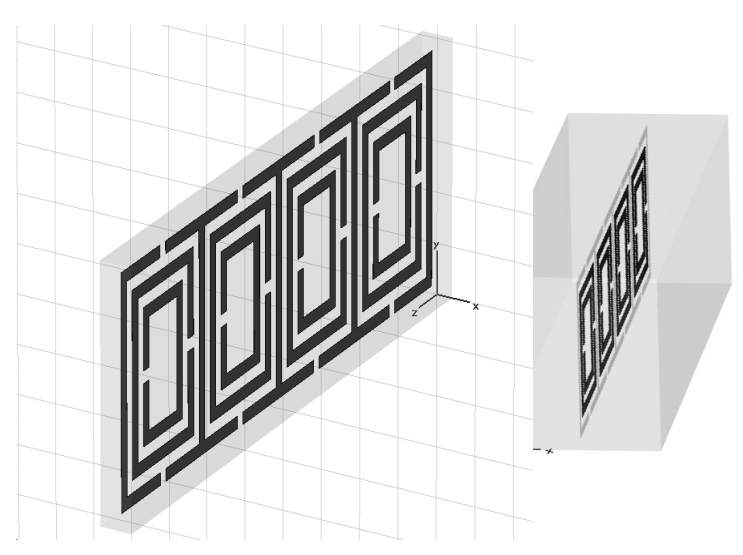

Figure 2(a) geometry of split ring and capacitive loaded strips

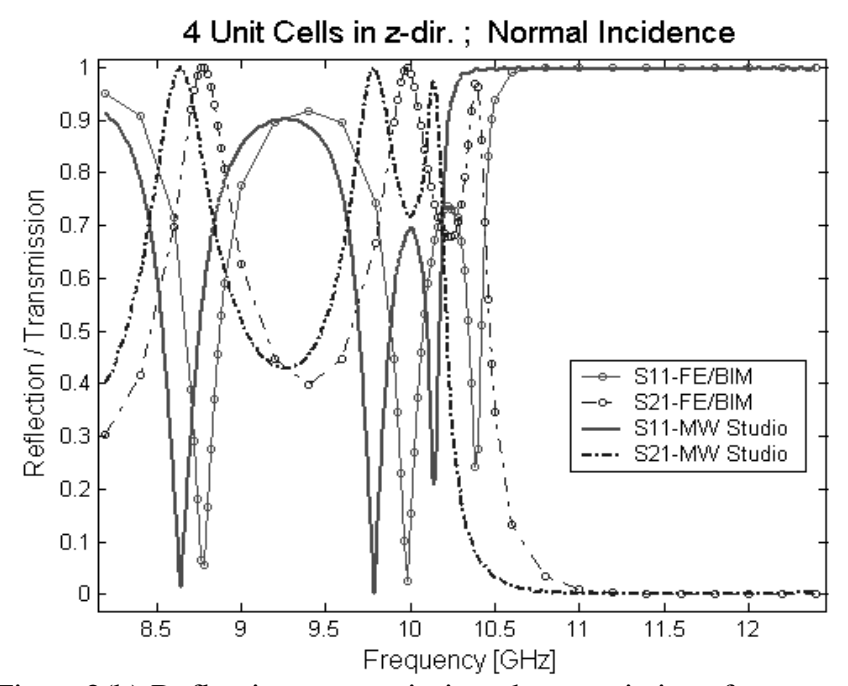

Figure2(b) Reflection, transmission characteristics of split ring/strip geometry

\section{Extraction of Electrical Properties:}

Following the Nicholson-Ross [4] method to estimate electric and magnetic properties of meta-materials, it is assumed that the dielectric slab with the metallic inclusions is replaced by a homogeneous material slab having effective permittivity $\left(\varepsilon_{r}^{e f f}\right)$ and permeability $\left(\mu_{r}^{e f f}\right)$. The reflection and transmission coefficients of this effective slab can be written as

$$
\begin{gathered}
S_{11}=r\left\{\left(1-t^{2}\right) /\left(1-r^{2} t^{2}\right)\right\}, \quad S_{21}=\left(\left(1-r^{2}\right) t\right) /\left(1-r^{2} t^{2}\right) \\
\text { where } t=e^{-j k_{0} d \sqrt{\left(\mu_{r}^{e f f} \varepsilon_{r}^{e f f}\right)}} \text { and } r=\left(\sqrt{\mu_{r}^{e f f} / \varepsilon_{r}^{e f f}}-1\right) /\left(\sqrt{\mu_{r}^{e f f} / \varepsilon_{r}^{e f f}}+1\right) \text {. The problem of extraction of electric }
\end{gathered}
$$

properties of an equivalent slab can now be defined as determination of $\varepsilon_{r}^{e f f}, \mu_{r}^{e f f}$ using (3) from the knowledge of $S_{11}, S_{21}, d$. Defining new variables $V_{1}=S_{11}+S_{21}, V_{2}=S_{21}-S_{11}$ and $X=\left(1-V_{1} V_{2}\right) /\left(V_{1}-V_{2}\right)$, it can be shown that

$$
r=X \pm \sqrt{X^{2}-1} \text { and } t=\left(V_{1}-r\right) /\left(1-V_{1} r\right)
$$

The steps involved in extraction process are:

(a) determine $(r, t)$ using (4),

(b) express $\sqrt{\mu_{r}^{\text {eff }} \varepsilon_{r}^{\text {eff }}}=k_{r}=\frac{1}{k_{0} d}\left(-\Phi_{t}-2 \pi n+j \ln (|t|)\right)$, where $n=\ldots . .-3,-2,-1,0,1,2,3, \ldots \ldots .$.

(c) with proper choice of n, parameters are given by $\mu_{r}^{\text {eff }}=((1+r) /(1-r)) k_{r}, \varepsilon_{r}^{\text {eff }}=((1-r) /(1+r)) k_{r}$

Due to ambiguity in selecting proper value of $\mathrm{n}$, earlier researchers [7] assuming $d \ll \lambda$ had simplified the calculations in the step (b). However, the assumption $d \ll \lambda$ limits application to very thin slab. In this work we have devel- 
oped a procedure to track correct value of $n$ without making the assumption. The proper values of effective electric properties are also estimated using the Genetic Algorithm.

\section{Numerical Results}

In this section numerical data for the effective parameters of a slab embedded with split rings arranged in a periodic fashion, shown in Figure 3(a), are presented. First, the unit cell including a single layer of split ring is modeled using a CAD package. The transmission and reflection coefficients as a function of frequency are then computed using the CST Microwave Studio software. Using the extraction procedure described above the effective parameters are estimated and are shown in Figure 3(b). Using the extracted parameters the reflection and transmission coefficients are calculated and compared with the numerical data obtained using the CST Microwave Studio.

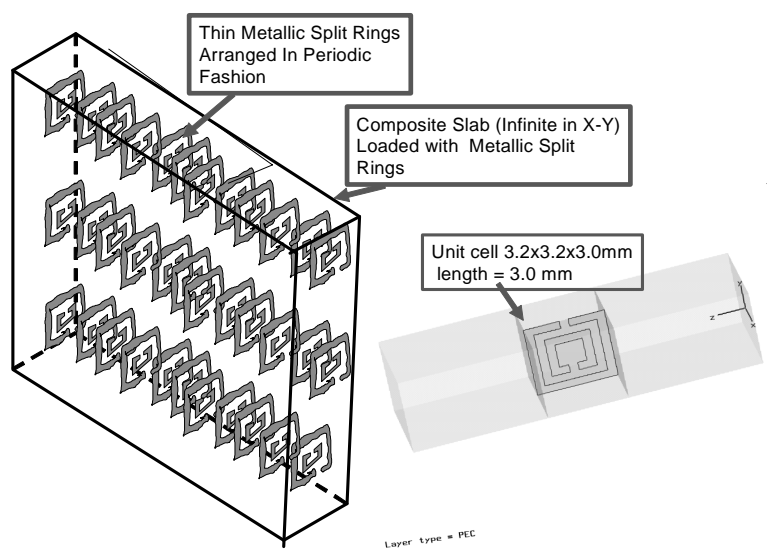

Figure 3(a) Geometry of a slab loaded with split rings

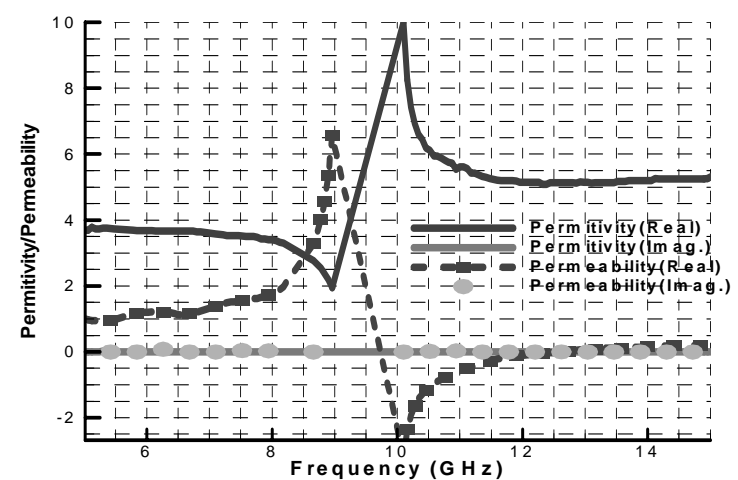

Figure 3(b) Effective parameters of slab loaded with split rings.

\section{Conclusions}

A computational electromagnetic method using the FEM and FDTD approaches have been presented to estimate the reflection and transmission through a meta-material slab. Nicholson-Ross technique[4] and also Genetic Algorithm procedure in conjunction with the knowledge of reflection and transmission coefficients have been used to extract effective permittivity and permeability of a meta-material slab. The reflection and transmission coefficient of actual meta-material slab have compared well with the numerical data obtained using the extracted effective permittivity and permeability. Due to page limitation the numerical results for a metallic inclusion in the shape of split ring only has been shown here. The metallic inclusions of other shapes will be discussed at the time of presentation.

\section{References}

[1] P. Markos and C. M. Soukoulis, "Transmission studies of left-handed materials," Physical Review B, Volume 65, December 7, 2001, 033401, pp. 033401-1 033401-4

[2] R. A. Shelby and et. al., "Experimental verification of a negative index of refraction," Science, Vol. 292, pp. 7779, Apr. 2001

[3] D. R. Smith and et. al., "Determination of negative permittivity and permeability of meta-materials from reflection and transmission coefficients," Phy. Rev. B, Vol. 65, pp. 195104(1)-19510(4), April 2002

[4] A. M. Nicholson and G.F. Ross, "Measurement of the intrinsic properties of materials by time domain techniques, ” IEEE Trans. Instrum. Mea., Vol. IM 17, pp. 395-402, Dec. 1968.

[5] Manohar D. Deshpande and et al., "A new approach to estimate complex permittivity of dielectric materials at microwave frequencies using waveguide measurements," IEEE Trans. on Microwave Theory and Techniques, Vol. MTT-45, No. 3, pop 359-366, March 1997

[6] “CST Microwave Studio, HF Design and Analysis," CST -Computer Simulation Technology, www.cst.com

[7] R. W. ZIolkowski, “ Design, fabrication and testing of double negative meta-materials, ” IEEE Trans. Antennas and Propagation, Vol. 51, pp. 1516-1529, July 2003 